\title{
Color-Kinematics Duality and the Regge Limit of Inelastic Amplitudes
}

\author{
Agustín Sabio Vera ${ }^{a}{ }_{1}^{1}$, Eduardo Serna Campillob 2 , Miguel Á. Vázquez-Mozo $\left.0^{b, a}\right]_{3}$ \\ ${ }^{a}$ Instituto de Física Teórica UAM/CSIC \& Universidad Autónoma de Madrid \\ C/ Nicolás Cabrera 15, E-28049 Madrid, Spain \\ ${ }^{b}$ Departamento de Física Fundamental \& IUFFyM, Universidad de Salamanca \\ Plaza de la Merced s/n, E-37008 Salamanca, Spain
}

\begin{abstract}
We investigate tree-level five-point amplitudes in scalar-QCD expressed in terms of Sudakov variables and find the equivalent "gravitational" counterparts using the color-kinematics duality proposed by Bern, Carrasco, and Johannson. Taking the multi-Regge limit in the gravitational amplitudes, we show that those pieces in the coupling of two reggeized gravitons to one on-shell graviton directly stemming from the double copy of the vertex for two reggeized gluons to one on-shell gluon are universal and properly reproduced by the duality.
\end{abstract}

\footnotetext{
${ }^{1}$ a.sabio.vera@gmail.com

${ }^{2}$ eduardo.serna@usal.es

${ }^{3}$ Miguel.Vazquez-Mozo@cern.ch
} 


\section{Introduction}

The relation between scattering amplitudes in gauge and gravity theories has been the subject of extensive research in recent years (for reviews on the subject we refer the reader to [1]). The fact that in a certain sense gravity can be regarded as the "square" of a gauge theory is suggested by string theory where tree-level graviton (closed string) amplitudes can be written as linear combinations of the product of gluon (open string) amplitudes, as found by Kawai, Lewellen, and Tye (KLT) [2]. In the field theory limit they translate into similar relations for the corresponding graviton and gluon amplitudes in quantum field theory [3, 4, 5]. Unfortunately, no similar factorization is known for string loop amplitudes.

More recently, a surprising relation between gluon and graviton amplitudes, dubbed colorkinematics duality, has been found by Bern, Carrasco, and Johansson (BCJ) [6] (see also [7, 8, 9, 10, 11]). The tree-level $n$-gluon amplitudes have the general structure

$$
\mathcal{A}(1, \ldots, n)_{\text {tree }}=g^{n-2} \sum_{i \in \Gamma} \frac{C_{i} N_{i}}{\prod_{\alpha} s_{\alpha}},
$$

where the sum runs over all Feynman diagram topologies $\Gamma, C_{i}$ are their color factors, $N_{i}$ the corresponding numerators, and $s_{\alpha}$ the kinematic invariants associated to the internal propagators of each diagram. Choosing the phases of the color factors properly, they satisfy Jacobi identities of the form

$$
C_{i}+C_{j}+C_{k}=0
$$

for certain triplets of indices $(i, j, k)$.

The amplitude (1.1) is invariant under generalized gauge transformations [6, 8] shifting the numerators in the form $N_{i} \rightarrow N_{i}+\Delta_{i}$, where $\Delta_{i}$ are functions of the momenta satisfying

$$
\sum_{i \in T} \frac{C_{i} \Delta_{i}}{\prod_{\alpha} s_{\alpha}}=0 .
$$

These are generalizations of the standard gauge transformations shifting the gluon polarization vectors $\varepsilon_{\mu}(k) \rightarrow \varepsilon_{\mu}(k)+\lambda k_{\mu}$, with $\lambda$ being a function of the momenta. This new freedom can be used to choose the numerators $N_{i}$ such that they replicate the Jacobi identities of the corresponding color factors shown in Eq. (1.2), namely

$$
N_{i}+N_{j}+N_{k}=0,
$$


for the same triplets $(i, j, k)$. The numerators obtained by the direct application of the Feynman rules in QCD do not fulfill these BCJ duality relations. In Ref. [8] a nonlocal action for the gluon field was constructed whose Feynman rules give numerators satisfying (1.4) for the five gluon tree-level amplitude.

Color-kinematics duality provides a prescription to construct the amplitude for the tree-level scattering of $n$ gravitons from two copies of the tree-level amplitude of $n$ gluons as [6]

$$
-i \mathcal{M}(1, \ldots, n)_{\text {tree }}=\left(\frac{\kappa}{2}\right)^{n-2} \sum_{i \in \Gamma} \frac{N_{i} \widetilde{N}_{i}}{\prod_{\alpha} s_{\alpha}} .
$$

Here $N_{i}, \widetilde{N}_{i}$ are two replicas of the numerators of the gauge theory amplitude (1.1) satisfying the BCJ duality relations (1.4) and $\kappa$ the gravitational coupling. This hidden connection between gauge theories and gravity amplitudes is very remarkable since, unlike the KLT relations, it is expected to hold also for loop amplitudes before integration over the loop momenta.

Whenever possible it is important to test the validity of this intriguing duality. Different kinematic limits of scattering amplitudes either in gauge or gravitational amplitudes are known which can serve as a test ground for the BCJ procedure. One recent example is the soft limit investigated in [12]. In the present work we focus on the Regge limit, which is well understood both in the gauge [13, 14, 15] and gravitational sides [16, 17, 18, 19, 20, 21]. At loop level, $\mathcal{N}$-supergravity amplitudes have been constructed from those of Yang-Mills theories even in the case of non-maximally supersymmetric theories [22]. In [20] it has been shown that the four-graviton amplitudes at two loops calculated using the BCJ duality for $\mathcal{N} \geq 4$ generate the correct Regge limit even at double logarithmic (in energy) accuracy, which goes beyond the well-known exponentiation of infrared divergencies. In the same work predictions have been made for graviton scattering in all supergravities and Einstein-Hilbert gravity to arbitrary order in the gravitational coupling which should serve as a good test of the color-kinematics duality at higher orders.

In Ref. 21] we carried out a study of exact inelastic amplitudes both in QCD and EinsteinHilbert gravity. Using a representation in Sudakov variables, we were able to reproduce the results of Lipatov for the emission of a graviton in multi-Regge kinematics (MRK). It turns out that this limit can be related to that of gluon production in QCD and an interesting double-copy structure emerges, which we investigate in the present work at the light of the color-kinematics 
duality. Here we find it useful to investigate the color-kinematics duality in scalar QCD (sQCD) by studying the scattering of two distinct scalars with the associated emission of a gluon in MRK, in this way it is possible to understand which pieces are somehow universal in the Regge limit and which ones are dependent on the matter content of the gauge theory when applying the BCJ procedure.

\section{2 sQCD and color-kinematics duality}

Our starting point is the tree-level contribution to the scattering of two distinguishable massless scalar particles with the emission of one gluon in sQCD

$$
\Phi(p, j)+\Phi^{\prime}(q, m) \longrightarrow \Phi\left(p^{\prime}, i\right)+\Phi\left(q^{\prime}, i\right)+g(k, a, \varepsilon)
$$

where in brackets we indicated the momenta and color quantum numbers of the involved particles, as well as the polarization vector in the case of the gluon. We choose this amplitude in order to reduce the number of diagrams to be calculated and to understand how the BCJ procedure fails when in the external states we not only have gluons but also scalar fields. The amplitude receives contributions from the following seven diagrams

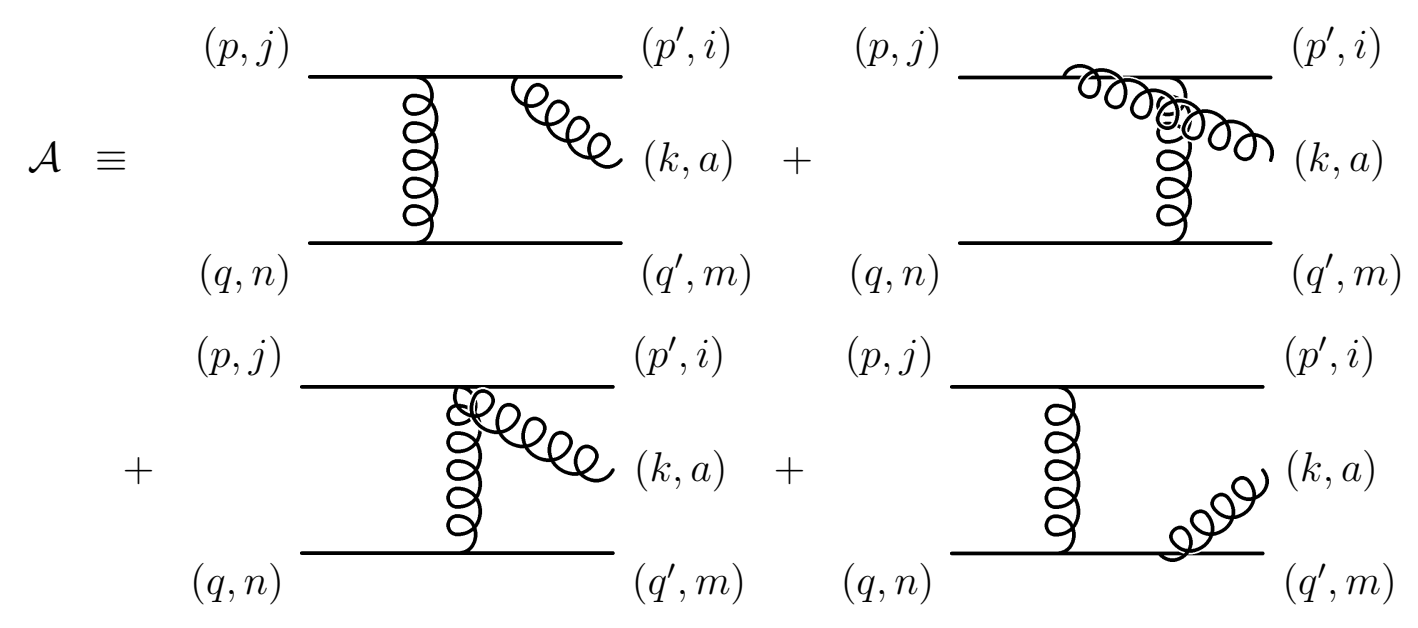




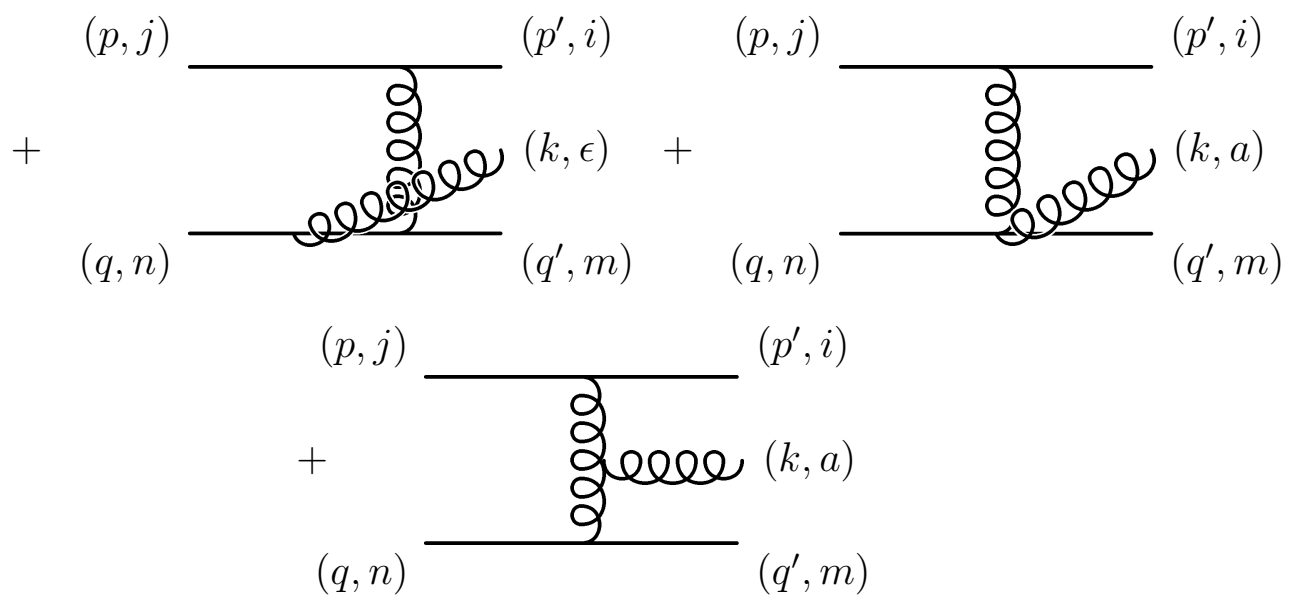

and has the general structure

$$
\mathcal{A}=g^{3}\left(\frac{C_{1} N_{1}}{t^{\prime} s_{p^{\prime} k}}+\frac{C_{2} N_{2}}{t^{\prime} s_{p k}}+\frac{C_{3} N_{3}}{t^{\prime}}+\frac{C_{4} N_{4}}{t s_{q^{\prime} k}}+\frac{C_{5} N_{5}}{t s_{q k}}+\frac{C_{6} N_{6}}{t}+\frac{C_{7} N_{7}}{t t^{\prime}}\right),
$$

where the numbering corresponds to the order in which the diagrams appear in Eq. (2.2). In writing the amplitude we have introduced the following kinematic invariants

$$
\begin{aligned}
t & =\left(p-p^{\prime}\right)^{2}, \\
t^{\prime} & =\left(q-q^{\prime}\right)^{2}, \\
s_{p k} & =(p+k)^{2}, \\
s_{p^{\prime} k} & =\left(p^{\prime}+k\right)^{2}, \\
s_{q k} & =(q+k)^{2}, \\
s_{q^{\prime} k} & =\left(q^{\prime}+k\right)^{2},
\end{aligned}
$$


and color factors

$$
\begin{aligned}
& C_{1}=T_{i k}^{a} T_{k j}^{b} T_{m n}^{b}, \\
& C_{2}=T_{i k}^{b} T_{k j}^{a} T_{m n}^{b} \\
& C_{3}=T_{i k}^{a} T_{k j}^{b} T_{m n}^{b}+T_{i k}^{b} T_{k j}^{a} T_{m n}^{b} \\
& C_{4}=T_{i j}^{b} T_{m k}^{a} T_{k n}^{b}, \\
& C_{5}=T_{i j}^{b} T_{m k}^{b} T_{k n}^{a} \\
& C_{6}=T_{i j}^{b} T_{m k}^{a} T_{k n}^{b}+T_{i j}^{b} T_{m k}^{b} T_{k n}^{a}, \\
& C_{7}=i f^{a b c} T_{i j}^{b} T_{m n}^{c}
\end{aligned}
$$

with $T_{i j}^{a}$ being the generators of the representation in which the scalar fields transform. They satisfy the Jacobi-like identities

$$
\begin{aligned}
& C_{1}-C_{2}+C_{7}=0, \\
& C_{1}+C_{2}-C_{3}=0, \\
& C_{4}-C_{5}-C_{7}=0, \\
& C_{4}+C_{5}-C_{6}=0 .
\end{aligned}
$$

Applying the Feynman rules 1 of sQCD, we find the following form for the numerators $N_{i}$

$$
\begin{aligned}
N_{1} & =2 i\left[\left(p+p^{\prime}+k\right) \cdot\left(q+q^{\prime}\right)\right]\left[p^{\prime} \cdot \varepsilon(k)\right], \\
N_{2} & =-2 i\left[\left(p+p^{\prime}-k\right) \cdot\left(q+q^{\prime}\right)\right][p \cdot \varepsilon(k)], \\
N_{3} & =-i\left(q+q^{\prime}\right) \cdot \varepsilon(k), \\
N_{4} & =2 i\left[\left(q+q^{\prime}+k\right) \cdot\left(p+p^{\prime}\right)\right]\left[q^{\prime} \cdot \varepsilon(k)\right], \\
N_{5} & =-2 i\left[\left(q+q^{\prime}-k\right) \cdot\left(p+p^{\prime}\right)\right][q \cdot \varepsilon(k)], \\
N_{6} & =-i\left(p+p^{\prime}\right) \cdot \varepsilon(k), \\
N_{7} & =-i\left\{\left[\left(q+q^{\prime}\right) \cdot\left(p-p^{\prime}+k\right)\right]\left[\left(p+p^{\prime}\right) \cdot \varepsilon(k)\right]\right. \\
& +\left[\left(p+p^{\prime}\right) \cdot\left(q+q^{\prime}\right)\right]\left[\left(p^{\prime}-p-q^{\prime}+q\right) \cdot \varepsilon(k)\right] \\
& \left.+\left[\left(p+p^{\prime}\right) \cdot\left(q^{\prime}-q-k\right)\right]\left[\left(q+q^{\prime}\right) \cdot \varepsilon(k)\right]\right\} .
\end{aligned}
$$

\footnotetext{
${ }^{1}$ See, e.g., 23 . Notice, however, that in the conventions of this reference the gauge group generators are anti-Hermitian, whereas we take them Hermitian.
} 
These numerators do not satisfy the BCJ duality relations derived from Eq. (2.6) and are therefore not ready to apply the color-kinematic duality prescription. The generalized nonlocal gauge transformation

$$
\begin{aligned}
& N_{1}^{\prime}=N_{1}+s_{p^{\prime} k}\left(N_{3}-\frac{N_{7}}{2 t}\right), \\
& N_{2}^{\prime}=N_{2}+s_{p k}\left(N_{3}+\frac{N_{7}}{2 t}\right), \\
& N_{3}^{\prime}=N_{3}, \\
& N_{4}^{\prime}=N_{4}+s_{q^{\prime} k}\left(N_{6}+\frac{N_{7}}{2 t^{\prime}}\right), \\
& N_{5}^{\prime}=N_{5}+s_{q k}\left(N_{6}-\frac{N_{7}}{2 t^{\prime}}\right), \\
& N_{6}^{\prime}=N_{6}, \\
& N_{7}^{\prime}=N_{7},
\end{aligned}
$$

recasts the amplitude in terms of only four color factors

$$
\mathcal{A}=g^{3}\left(\frac{C_{1} N_{1}^{\prime}}{t^{\prime} s_{p^{\prime} k}}+\frac{C_{2} N_{2}^{\prime}}{t^{\prime} s_{p k}}+\frac{C_{4} N_{4}^{\prime}}{t s_{q^{\prime} k}}+\frac{C_{5} N_{5}^{\prime}}{t s_{q k}}\right),
$$

satisfying the single Jacobi identity [derived from Eq. (2.6)]

$$
C_{1}-C_{2}+C_{4}-C_{5}=0 .
$$

These new numerators $N_{i}^{\prime}$ do not comply with BCJ duality. To fix this we perform a further generalized gauge transformation of the form

$$
\begin{aligned}
& N_{1}^{\prime \prime}=N_{1}^{\prime}+\alpha t^{\prime} s_{p^{\prime} k}, \\
& N_{2}^{\prime \prime}=N_{2}^{\prime}-\alpha t^{\prime} s_{p k}, \\
& N_{4}^{\prime \prime}=N_{4}+\alpha t s_{q^{\prime} k}, \\
& N_{5}^{\prime \prime}=N_{5}-\alpha t s_{q k},
\end{aligned}
$$

where the function $\alpha$ is determined by requiring

$$
N_{1}^{\prime \prime}-N_{2}^{\prime \prime}+N_{3}^{\prime \prime}-N_{4}^{\prime \prime}=0 .
$$


This gives

$$
\alpha=\frac{-N_{1}^{\prime}+N_{2}^{\prime}-N_{4}^{\prime}+N_{5}^{\prime}}{t^{\prime}\left(s_{p k}+s_{p^{\prime} k}\right)+t\left(s_{q k}+s_{q^{\prime} k}\right)} .
$$

After all these algebraic manipulations we have managed to write our amplitude in the form

$$
\mathcal{A}=g^{3}\left(\frac{C_{1} N_{1}^{\prime \prime}}{t^{\prime} s_{p^{\prime} k}}+\frac{C_{2} N_{2}^{\prime \prime}}{t^{\prime} s_{p k}}+\frac{C_{4} N_{4}^{\prime \prime}}{t s_{q^{\prime} k}}+\frac{C_{5} N_{5}^{\prime \prime}}{t s_{q k}}\right),
$$

where the numerators satisfy (2.12). The next step is to apply the color-kinematics duality prescription to construct the gravitational amplitude, i.e.

$$
-i \mathcal{M}=\left(\frac{\kappa}{2}\right)^{3}\left(\frac{N_{1}^{\prime \prime} \tilde{N}_{1}^{\prime \prime}}{t^{\prime} s_{p^{\prime} k}}+\frac{N_{2}^{\prime \prime} \tilde{N}_{2}^{\prime \prime}}{t^{\prime} s_{p k}}+\frac{N_{4}^{\prime \prime} \tilde{N}_{4}^{\prime \prime}}{t s_{q^{\prime} k}}+\frac{N_{5}^{\prime \prime} \tilde{N}_{5}^{\prime \prime}}{t s_{q k}}\right) .
$$

The graviton polarization tensor $\epsilon_{\mu \nu}(k)$ is reconstructed as

$$
\varepsilon_{\mu}(k) \widetilde{\varepsilon}_{\nu}(k) \longrightarrow \epsilon_{\mu \nu}(k)
$$

with $\varepsilon_{\mu}(k), \widetilde{\varepsilon}_{\mu}(k)$ being the gluon polarization vectors contained in the numerators $N_{i}^{\prime \prime}$ and $\widetilde{N}_{i}^{\prime \prime}$.

At this point it is convenient to redefine the momenta according to

$$
p^{\prime}=p-k_{1}, \quad q^{\prime}=q+k_{2}, \quad k=k_{1}-k_{2}
$$

and write $k_{1}$ and $k_{2}$ using Sudakov parameters

$$
k_{1}^{\mu}=\alpha_{1} p^{\mu}+\beta_{1} q^{\mu}+k_{1, \perp}^{\mu}, \quad k_{2}^{\mu}=\alpha_{2} p^{\mu}+\beta_{2} q^{\mu}+k_{2, \perp}^{\mu} .
$$

In this representation for the momenta, the amplitude

$$
-i \mathcal{M} \equiv-i A_{k k} \mathcal{M}^{\mu \nu} \epsilon_{\mu \nu}(k)
$$

can be shown to have the following tensor structure

$$
\begin{aligned}
\mathcal{M}^{\mu \nu} & =\left(k_{1}+k_{2}\right)_{\perp}^{\mu}\left(k_{1}+k_{2}\right)_{\perp}^{\nu}+\mathcal{A}_{k p}\left[\left(k_{1}+k_{2}\right)_{\perp}^{\mu} p^{\nu}+p^{\mu}\left(k_{1}+k_{2}\right)_{\perp}^{\nu}\right] \\
& +\mathcal{A}_{k q}\left[\left(k_{1}+k_{2}\right)_{\perp}^{\mu} q^{\nu}+q^{\mu}\left(k_{1}+k_{2}\right)_{\perp}^{\nu}\right]+\mathcal{A}_{p q}\left(p^{\mu} q^{\nu}+q^{\mu} p^{\nu}\right) \\
& +\mathcal{A}_{q q} q^{\mu} q^{\nu}+\mathcal{A}_{p p} p^{\mu} p^{\nu} .
\end{aligned}
$$


Let us point out that in Eq. (2.19) we have factored out the coefficient $A_{k k}$ of the term proportional to $\left(k_{1}+k_{2}\right)_{\perp}^{\mu}\left(k_{1}+k_{2}\right)_{\perp}^{\nu}$. In the MRK limit

$$
1 \gg \alpha_{1} \gg \alpha_{2}, \quad 1 \gg\left|\beta_{2}\right| \gg\left|\beta_{1}\right|,
$$

the prefactors $\mathcal{A}_{i}$ have the following form

$$
\begin{aligned}
& \mathcal{A}_{p p}=\left(\alpha_{1}-2 \frac{\beta_{1}}{\beta_{2}}\right)^{2}+2 \alpha_{2} \beta_{1}\left(\frac{\alpha_{1}+\beta_{2}}{\beta_{2}^{2}}\right)+\ldots, \\
& \mathcal{A}_{q q}=\left(\beta_{2}+2 \frac{\alpha_{2}}{\alpha_{1}}\right)^{2}-2 \alpha_{2} \beta_{1}\left(\frac{\alpha_{1}+\beta_{2}}{\alpha_{1}^{2}}\right)+\ldots, \\
& \mathcal{A}_{p q}=\left(\alpha_{1}-2 \frac{\beta_{1}}{\beta_{2}}\right)\left(\beta_{2}+2 \frac{\alpha_{2}}{\alpha_{1}}\right)+\ldots, \\
& \mathcal{A}_{k p}=-\left(\alpha_{1}-2 \frac{\beta_{1}}{\beta_{2}}\right)+\ldots, \\
& \mathcal{A}_{k q}=-\left(\beta_{2}+2 \frac{\alpha_{2}}{\alpha_{1}}\right)+\ldots
\end{aligned}
$$

We want to compare these results, obtained after applying the BCJ color-kinematics duality, with the ones found in Ref. 21] using traditional Feynman rules for the gravitational scattering of two different scalars with emission of a graviton:

$$
\Phi(p)+\Phi^{\prime}(q) \longrightarrow \Phi\left(p^{\prime}\right)+\Phi^{\prime}\left(q^{\prime}\right)+G(k, \epsilon) .
$$

There we obtained that the tree-level amplitude in the MRK regime can be written as [in the notation of Eq. (2.19)]

$$
\mathcal{M}^{\mu \nu}=\Omega^{\mu} \Omega^{\nu}-\mathcal{N}^{\mu} \mathcal{N}^{\nu},
$$

where

$$
\Omega^{\mu}=\left(\alpha_{1}-\frac{2 \beta_{1}}{\beta_{2}}\right) p^{\mu}+\left(\beta_{2}+\frac{2 \alpha_{2}}{\alpha_{1}}\right) q^{\mu}-\left(k_{1}+k_{2}\right)_{\perp}^{\mu}
$$

is Lipatov's MRK effective vertex for the coupling of two reggeized gluons to an on-shell gluon in $\mathrm{QCD}$, and

$$
\mathcal{N}^{\mu}=-2 i \sqrt{\beta_{1} \alpha_{2}}\left(\frac{p^{\mu}}{\beta_{2}}+\frac{q^{\mu}}{\alpha_{1}}\right) .
$$


The term $\mathcal{N}^{\mu} \mathcal{N}^{\nu}$ in Eq. (2.24) is responsible for the cancellation of overlapping singularities in simultaneous channels required by the Steinmann relations [24] (see also [25, 26] for a recent discussion). It is important to realize that Eq. (2.22) does not reproduce the full structure indicated in Eq. (2.24). This is an effect of applying the color-kinematics duality to an amplitude where some of the external states are not gluons in the gauge theory side. However, we can see that the term corresponding purely to the "square" of the gauge vertex, $\Omega^{\mu} \Omega^{\nu}$, is correctly reproduced by the BCJ prescription in the Regge limit.

In order to show that, although the $\mathcal{N}^{\mu} \mathcal{N}^{\nu}$ terms are not given by BCJ color-kinematic duality, the $\Omega^{\mu} \Omega^{\nu}$ contributions are indeed retrieved, we apply the prescription to a set of numerators satisfying different BCJ duality identities. Using the local generalized gauge transformation

$$
\begin{aligned}
N_{1}^{\prime} & =N_{1}+s_{p^{\prime} k} N_{3}, \\
N_{2}^{\prime} & =N_{2}+s_{p k} N_{3}, \\
N_{3}^{\prime} & =N_{3}, \\
N_{4}^{\prime} & =N_{4}+s_{q^{\prime} k} N_{6}, \\
N_{5}^{\prime} & =N_{5}+s_{q k} N_{6}, \\
N_{7}^{\prime} & =N_{7},
\end{aligned}
$$

the sQCD amplitude can be written in the form

$$
\mathcal{A}=g^{3}\left(\frac{C_{1} N_{1}^{\prime}}{t^{\prime} s_{p^{\prime} k}}+\frac{C_{2} N_{2}^{\prime}}{t^{\prime} s_{p k}}+\frac{C_{4} N_{4}^{\prime}}{t s_{q^{\prime} k}}+\frac{C_{5} N_{5}^{\prime}}{t s_{q k}}+\frac{C_{7} N_{7}^{\prime}}{t t^{\prime}}\right) .
$$

A further transformation

$$
\begin{aligned}
& N_{1}^{\prime \prime}=N_{1}^{\prime}+\alpha t^{\prime} s_{p^{\prime} k}, \\
& N_{2}^{\prime \prime}=N_{2}^{\prime}-\alpha t^{\prime} s_{p k}, \\
& N_{4}^{\prime \prime}=N_{4}^{\prime}-\beta t s_{q^{\prime} k}, \\
& N_{5}^{\prime \prime}=N_{5}^{\prime}+\beta t s_{q k}, \\
& N_{7}^{\prime \prime}=N_{7}^{\prime}+(\alpha+\beta) t t^{\prime},
\end{aligned}
$$

is performed, where $\alpha$ and $\beta$ are rational functions of the kinematic invariants fully determined by the condition that the numerators $N_{i}^{\prime \prime}$ satisfy BCJ duality

$$
N_{1}^{\prime \prime}-N_{2}^{\prime \prime}+N_{7}^{\prime \prime}=0, \quad N_{4}^{\prime \prime}-N_{5}^{\prime \prime}-N_{7}^{\prime \prime}=0 .
$$


We apply now color-kinematics duality to construct the amplitude:

$$
-i \mathcal{M}=\left(\frac{\kappa}{2}\right)^{3}\left(\frac{N_{1}^{\prime \prime} \tilde{N}_{1}^{\prime \prime}}{t^{\prime} s_{p^{\prime} k}}+\frac{N_{2}^{\prime \prime} \tilde{N}_{2}^{\prime \prime}}{t^{\prime} s_{p k}}+\frac{N_{4}^{\prime \prime} \tilde{N}_{2}^{\prime \prime}}{t s_{q^{\prime} k}}+\frac{N_{5}^{\prime \prime} \tilde{N}_{5}^{\prime \prime}}{t s_{q k}}+\frac{N_{7}^{\prime \prime} \tilde{N}_{7}^{\prime \prime}}{t t^{\prime}}\right),
$$

that has the structure given in Eqs. (2.19) and (2.20). The calculation of the coefficients $\mathcal{A}_{i}$ in MRK gives

$$
\begin{aligned}
\mathcal{A}_{p p} & =\left(\alpha_{1}-2 \frac{\beta_{1}}{\beta_{2}}\right)^{2}+4 \alpha_{2} \beta_{1}\left(\frac{\alpha_{1}}{\beta_{2}^{2}}\right)+\ldots, \\
\mathcal{A}_{q q} & =\left(\beta_{2}+2 \frac{\alpha_{2}}{\alpha_{1}}\right)^{2}-4 \alpha_{2} \beta_{1}\left(\frac{\beta_{2}}{\alpha_{1}^{2}}\right)+\ldots, \\
\mathcal{A}_{p q} & =\left(\alpha_{1}-2 \frac{\beta_{1}}{\beta_{2}}\right)\left(\beta_{2}+2 \frac{\alpha_{2}}{\alpha_{1}}\right)+\ldots, \\
\mathcal{A}_{k p} & =-\left(\alpha_{1}-2 \frac{\beta_{1}}{\beta_{2}}\right)+\ldots, \\
\mathcal{A}_{k q} & =-\left(\beta_{2}+2 \frac{\alpha_{2}}{\alpha_{1}}\right)+\ldots
\end{aligned}
$$

Again, this correctly reproduces the part $\Omega^{\mu} \Omega^{\nu}$ of the MRK gravitational amplitude, but it falls short of reproducing the terms canceling the overlapping divergences.

To conclude our analysis, we would like to investigate which topologies in the original Feynman diagrams contribute to the different terms in the coefficients obtained in Eq. (2.22). To trace each contribution, we rescale the original numerators in Eq. (2.7) by some constants $D_{i}$ according to $N_{i} \rightarrow N_{i} D_{i}$ and study the expansion in the MRK regime. This results in the following form for the coefficients:

$$
\begin{aligned}
\mathcal{A}_{p p}= & \alpha_{1}^{2}-2\left(\frac{D_{1}+D_{2}}{D_{7}}\right) \frac{\alpha_{1} \beta_{1}}{\beta_{2}}+2\left(\frac{D_{1}^{2}+D_{2}^{2}}{D_{7}^{2}}\right) \frac{\beta_{1}^{2}}{\beta_{2}^{2}}+\left(\frac{D_{1}+D_{2}}{D_{7}}\right) \frac{\alpha_{2} \beta_{1} \alpha_{1}}{\beta_{2}^{2}} \\
- & {\left[\frac{D_{1}^{2}-3 D_{2} D_{1}+3 D_{4} D_{1}+2 D_{2}^{2}+3 D_{2} D_{4}-4\left(D_{1}+D_{2}\right) D_{7}}{D_{7}^{2}}\right] \frac{\alpha_{2} \beta_{1}}{\beta_{2}}+\ldots } \\
& \longrightarrow\left(\alpha_{1}-2 \frac{D_{b}}{D_{7}} \frac{\beta_{1}}{\beta_{2}}\right)^{2}+\frac{\alpha_{2} \beta_{1}\left[2 D_{7} D_{b} \alpha_{1}-\beta_{2}\left(6 D_{b}^{2}-8 D_{7} D_{b}\right)\right]}{D_{7}^{2} \beta_{2}^{2}} .
\end{aligned}
$$

Note that, in the last line, we have simplified the expression collecting similar topologies using the same constant for them, i.e. $D_{b} \equiv D_{1}=D_{2}=D_{4}=D_{5}$, where with the subscript $b$ indicates 
that they are associated with the diagrams where the gluon is emitted by bremsstrahlung from a scalar line. $D_{3,6}$ mark the diagrams containing the scalar-scalar-gluon-gluon vertex and $D_{7}$ the diagram with the three gluon vertex. The remaining results are

$$
\begin{aligned}
& \mathcal{A}_{q q}=\beta_{2}^{2}+2\left(\frac{D_{4}+D_{5}}{D_{7}}\right) \frac{\alpha_{2} \beta_{2}}{\alpha_{1}}+2\left(\frac{D_{4}^{2}+D_{5}^{2}}{D_{7}^{2}}\right) \frac{\alpha_{2}^{2}}{\alpha_{1}^{2}}-\left(\frac{D_{4}+D_{5}}{D_{7}}\right) \frac{\alpha_{2} \beta_{1} \beta_{2}}{\alpha_{1}^{2}}+\ldots \\
& +\left[\frac{D_{4}^{2}-3 D_{5} D_{4}+2 D_{5}^{2}+\left(D_{4}+D_{5}\right)\left(3 D_{1}-4 D_{7}\right)}{D_{7}^{2}}\right] \frac{\alpha_{2} \beta_{1}}{\alpha_{1}} \\
& \longrightarrow\left(\beta_{2}+2 \frac{D_{b}}{D_{7}} \frac{\alpha_{2}}{\alpha_{1}}\right)^{2}+\frac{\alpha_{2} \beta_{1}\left(\alpha_{1}\left(6 D_{b}^{2}-8 D_{7} D_{b}\right)-2 D_{7} \beta_{2} D_{b}\right)}{D_{7}^{2} \alpha_{1}^{2}}, \\
& \mathcal{A}_{p q}=\alpha_{1} \beta_{2}-\frac{\left(D_{1}+D_{2}\right)}{D_{7}} \beta_{1}+\frac{\left(D_{4}+D_{5}\right) \alpha_{2}}{D_{7}}-\frac{\left(D_{1}+D_{2}\right)\left(D_{4}+D_{5}\right)}{D_{7}^{2}} \frac{\beta_{1} \alpha_{2}}{\alpha_{1} \beta_{2}}+\ldots \\
& \longrightarrow\left(\alpha_{1}-2 \frac{D_{b}}{D_{7}} \frac{\beta_{1}}{\beta_{2}}\right)\left(\beta_{2}+2 \frac{D_{b}}{D_{7}} \frac{\alpha_{2}}{\alpha_{1}}\right) \\
& \mathcal{A}_{k p}=-\alpha_{1}+\frac{\left(D_{1}+D_{2}\right)}{D_{7}} \frac{\beta_{1}}{\beta_{2}}+\ldots \\
& \longrightarrow-\left(\alpha_{1}-2 \frac{D_{b}}{D_{7}} \frac{\beta_{1}}{\beta_{2}}\right) \\
& \mathcal{A}_{k q}=-\beta_{2}-\frac{\left(D_{4}+D_{5}\right)}{D_{7}} \frac{\alpha_{2}}{\alpha_{1}}+\ldots \\
& \longrightarrow-\left(\beta_{2}+2 \frac{D_{b}}{D_{7}} \frac{\alpha_{2}}{\alpha_{1}}\right), \\
& \mathcal{A}_{k q}=-\beta_{2}-\frac{\left(D_{4}+D_{5}\right)}{D_{7}} \frac{\alpha_{2}}{\alpha_{1}}+\ldots \\
& \longrightarrow \quad-\left(\beta_{2}+2 \frac{D_{b}}{D_{7}} \frac{\alpha_{2}}{\alpha_{1}}\right) .
\end{aligned}
$$

It is remarkable that the MRK limit is blind to the constants $D_{3,6}$ and therefore to the diagrams with the scalar-scalar-gluon-gluon vertex. 


\section{Discussion}

We have investigated the color-kinematics duality proposed by Bern, Carrasco, and Johannson for the construction of gravitational amplitudes as a formal double-copy of equivalent amplitudes in gauge theories. In the gauge theory side we work with scalar QCD, and study the scattering of two distinct scalar with production of a gluon in the final state. We write the amplitude in two different representations and construct the corresponding "gravitational" scattering amplitudes using the BCJ doubling prescription. Despite them being different, when taking the multi-Regge limit of these amplitudes both BCJ representations reproduce the part of the MRK gravitational vertex (with two reggeized gravitons and one on-shell graviton) which corresponds to the square of the MRK vertex in QCD (with two reggeized gluons and one onshell gluon). The subleading terms responsible for the fulfillment of the Steinmann conditions are not reproduced correctly and are dependent on the choice of numerators satisfying BCJ duality used to write the gauge amplitude. This has a likely origin in the external matter states of the gauge amplitudes, for which the duality does not hold.

\section{Acknowledgments}

We thank Henrik Johansson for a useful discussion during the 2012 Informal Meeting on Scattering Amplitudes \& the Multi-Regge limit, at the Instituto de Física Teórica UAM/CSIC. ASV acknowledges partial support from the European Comission under contract LHCPhenoNet (PITN-GA-2010-264564), the Madrid Regional Government through Proyecto HEPHACOS ESP-1473, the Spanish Government MICINN (FPA2010-17747) and Spanish MINECOs "Centro de Excelencia Severo Ochoa" Programme under grant SEV-2012-0249. The work of ESC has been supported by a Spanish Government FPI Predoctoral Fellowship and grant FIS2009-07238. MAVM acknowledges partial support from Spanish Government grants FPA2009-10612 and FIS2009-07238, Basque Government Grant IT-357-07 and Spanish Consolider-Ingenio 2010 Programme CPAN (CSD2007-00042). MAVM thanks the Instituto de Física Teórica UAM/CSIC for kind hospitality. 


\section{References}

[1] Z. Bern, Perturbative quantum gravity and its relation to gauge theory, Living Rev. Rel. 5 (2002) 5 [gr-qc/0206071] .

J. J. M. Carrasco and H. Johansson, Generic multiloop methods and application to $\mathcal{N}=4$ super-Yang-Mills, J. Phys. A44 (2011) 454004 [arXiv:1103.3298 [hep-th]].

[2] H. Kawai, D. C. Lewellen and S. H. H. Tye, A Relation Between Tree Amplitudes of Closed and Open Strings, Nucl. Phys. B269 (1986) 1.

[3] D. C. Dunbar, P. S. Norridge, Calculation of graviton scattering amplitudes using string based methods, Nucl. Phys. B433 (1995) 181. [arXiv:hep-th/9408014] .

[4] Z. Bern, D. C. Dunbar, T. Shimada, String based methods in perturbative gravity, Phys. Lett. B312 (1993) 277. [arXiv:hep-th/9307001] .

[5] Z. Bern, D. C. Dunbar, A Mapping between Feynman and string motivated one loop rules in gauge theories, Nucl. Phys. B379 (1992) 562.

[6] Z. Bern, J. J. M. Carrasco and H. Johansson, New Relations for Gauge-Theory Amplitudes, Phys. Rev. D78 (2008) 085011 [arXiv:0805.3993 [hep-ph]].

[7] Z. Bern, J. J. M. Carrasco, H. Johansson, Perturbative Quantum Gravity as a Double Copy of Gauge Theory, Phys. Rev. Lett. 105 (2010) 061602. [arXiv:1004.0476 [hep-th]] .

[8] Z. Bern, T. Dennen, Y.-t. Huang and M. Kiermaier, Gravity as the Square of Gauge Theory, Phys. Rev. D82 (2010) 065003 [arXiv:1004.0693 [hep-th]].

[9] Z. Bern and T. Dennen, A Color Dual Form for Gauge-Theory Amplitudes, Phys. Rev. Lett. 107 (2011) 081601 [arXiv:1103.0312 [hep-th]] .

[10] S. H. Henry Tye and Y. Zhang, Dual Identities inside the Gluon and the Graviton Scattering Amplitudes, J. High Energy Phys. 06 (2010) 071 [Erratum-ibid. 04 (2011) 114] [arXiv:1003.1732 [hep-th]]. 
[11] C. R. Mafra, O. Schlotterer and S. Stieberger, Explicit BCJ Numerators from Pure Spinors, J. High Energy Phys. 07 (2011) 092 [arXiv:1104.5224 [hep-th]].

[12] S. Oxburgh and C. D. White, BCJ duality and the double copy in the soft limit, arXiv:1210.1110 [hep-th] .

[13] L. N. Lipatov, Reggeization of the Vector Meson and the Vacuum Singularity in Nonabelian Gauge Theories, Sov. J. Nucl. Phys. 23 (1976) 338.

[14] E. A. Kuraev, L. N. Lipatov, V. S. Fadin, On the Pomeranchuk Singularity in Asymptotically Free Theories, Phys. Lett. B 60 (1975) 50,

E. A. Kuraev, L. N. Lipatov, V. S. Fadin, Multi-Reggeon Processes in the Yang-Mills Theory, Sov. Phys. JETP 44 (1976) 443,

E. A. Kuraev, L. N. Lipatov, V. S. Fadin, The Pomeranchuk Singularity in Nonabelian Gauge Theories, Sov. Phys. JETP 45 (1977) 199.

[15] I. I. Balitsky, L. N. Lipatov, The Pomeranchuk Singularity in Quantum Chromodynamics, Sov. J. Nucl. Phys. 28 (1978) 822.

[16] L. N. Lipatov, Effective action for the Regge processes in gravity, arXiv:1105.3127 [hep-th] .

[17] L. N. Lipatov, Graviton Reggeization, Phys. Lett. B116 (1982) 411.

[18] L. N. Lipatov, Multi - Regge Processes In Gravitation, Sov. Phys. JETP 55 (1982) 582 [Zh. Eksp. Teor. Fiz. 82 (1982) 991].

[19] L. N. Lipatov, High-energy scattering in $Q C D$ and in quantum gravity and two-dimensional field theories, Nucl. Phys. B365 (1991) 614.

[20] J. Bartels, L. N. Lipatov and A. Sabio Vera, Double-logarithms in Einstein-Hilbert gravity and supergravity, arXiv:1208.3423 [hep-th] .

[21] A. Sabio Vera, E. Serna Campillo and M. A. Vázquez-Mozo, Graviton emission in EinsteinHilbert gravity, J. High Energy Phys 03 (2012) 005 [arXiv:1112.4494 [hep-th]] . 
[22] J. J. Carrasco and H. Johansson, Five-Point Amplitudes in N=4 Super-Yang-Mills Theory and $\mathcal{N}=8$ Supergravity, Phys. Rev. D85 (2012) 025006 [arXiv:1106.4711 [hep-th]] . Z. Bern, C. Boucher-Veronneau and H. Johansson, $\mathcal{N} \geq 4$ Supergravity Amplitudes from Gauge Theory at One Loop, Phys. Rev. D84 (2011) 105035 [arXiv:1107.1935 [hep-th] ].

[23] C. Itzykson and J. B. Zuber, Quantum Field Theory, McGraw-Hill 1980.

[24] O. Steinmann, Über den Zusammenhang zwischen den Wightmanfunktionen und der retardierten Kommutatoren, Helv. Phys. Acta 33 (1960) 257,

O. Steinmann, Wightman-Funktionen und retardierten Kommutatoren. II, Helv. Phys. Acta. 33 (1960) 347.

[25] J. Bartels, L. N. Lipatov, A. Sabio Vera, BFKL Pomeron, Reggeized gluons and Bern-Dixon-Smirnov amplitudes, Phys. Rev. D80 (2009) 045002. arXiv:0802.2065 [hep-th] ] .

[26] J. Bartels, L. N. Lipatov, A. Sabio Vera, $\mathcal{N}=4$ supersymmetric Yang Mills scattering amplitudes at high energies: The Regge cut contribution, Eur. Phys. J. C65 (2010) 587. [arXiv:0807.0894 [hep-th]]. 